\title{
Re-purposing Anticoagulation Clinics: Expanding Access to Opioid Agonist Therapy in Primary Care Settings
}

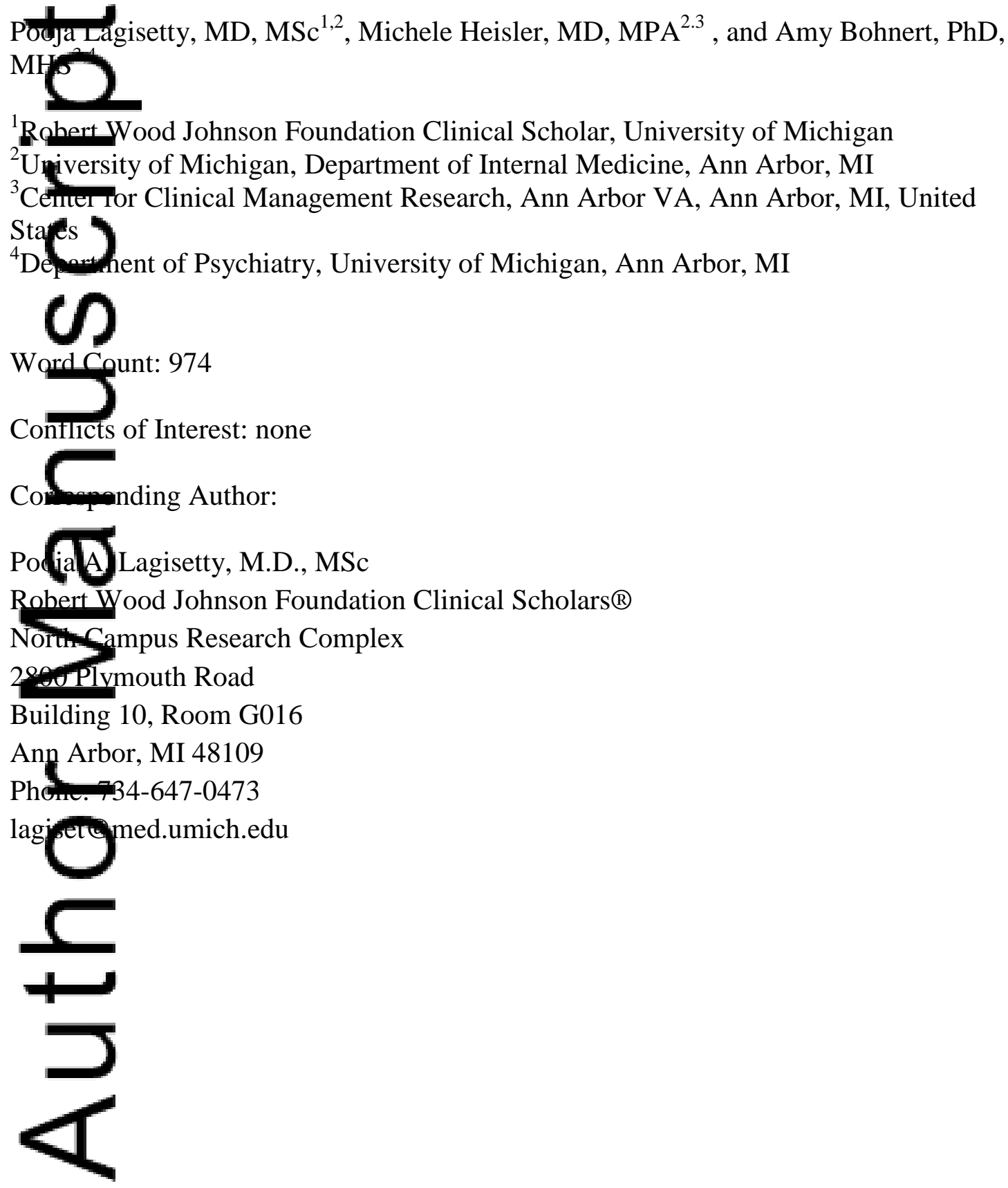

This is the author manuscript accepted for publication and has undergone full peer review but has not been through the copyediting, typesetting, pagination and proofreading process, which may lead to differences between this version and the Version of Record. Please cite this article as doi: $10.1111 /$ add.13531

This article is protected by copyright. All rights reserved. 
To overcome structural barriers to providing opioid agonist therapy $(O A T)$ in primary care settings, we propose building on the existing infrastructure of anticoagulation clinics. These clinics are well equipped to provide drug-monitoring and multidisciplinary support for medications through pharmacy and/or nurse-led teams. This proposal would ase resources to expand current anti-coagulation clinics' capabilities to include OAT.

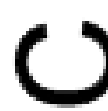

As pripry care physicians work to decrease opiate prescribing for chronic pain, more patients requiring treatment for opioid use disorders will be identified. Accordingly, policymakers internationally are calling to develop effective, scalable primary care mo increase access to therapies such as opioid agonist therapy (OAT). Globally, $\mathrm{mu}$ (t) Europe, Australia, and Canada have already been using general practitioners to proscribe not only buprenorphine, but also methadone $(1,2)$. The World Health Gigarreation has attested that in order to ensure the quality of OAT programs, care models in any setting need to follow national and international guidelines, be mulary environment, and be patient-centered (1). Primary care-based multidisciplinary models that deliyer OAT in the form of medication management clinics would meet these criteria $+$ whind so providing logistical support to an already overburdened primary care workforce. 
The necessary infrastructure for such medication management clinics already exists. Anticoagulation clinics, providing interdisciplinary medication management and mơhtoring for patients on warfarin, have been established in over 3,000 care delivery set matrnationally. Warfarin, similar to buprenorphine and methadone, requires fre uent lab monitoring, ongoing patient education, and protocols for dose adjustment. An anatgous OAT protocol design could easily account for the specific needs of patients during induction, maintenance, and stabilization phases of treatment. In addition, anticaagulation clinics are already well equipped to support both specialty and primary care physicians. Staffed by pharmacists, nurses, and/or nurse practitioners, these pro could conduct monitoring, patient education and counseling, and when autoliz d, implement dosing changes. This design would also be well suited to help support collaboration with multiple specialty providers, especially important when arratrate pain specialists do not have addiction expertise. In this collaborative care aproach, the nurses and pharmacists serve as liaisons between the primary care team and speramys. In addition, as newer direct oral anticoagulants that require less frequent laboratory monitoring are increasingly replacing warfarin for treatment of common cardiovascular conditions, anticoagulation clinics should have more capacity to start to mather medications. 
Re-purposing these clinics to safely manage multiple classes of drugs would reduce the start-up burden and cost of finding trained personnel and physical space to start OAT clinics. Moreover, by providing the familiarity of a parallel care structure and supports, pintary care physicians may be more likely to take on the challenge of providing buranine for patients with opioid addiction. Similarly, health care systems would be nore likely to invest in a previously tested model that provides high quality care and loyers gsts through reductions in hospitalizations and emergency department visits(3).

\section{$\infty$}

Logistical barriers are among the most commonly cited reasons by primary care physicians for not prescribing OAT(4). In addition, critics of increasing primary carebas scribing of OAT often express concern for drug safety. The tools of OAT safety inc udecking prescription drug monitoring programs, drug contracts, pill counts, and unmetoxicology screens to monitor for drug misuse and diversion. Medication rmarragentent clinics offer a solution to the burden of this monitoring, as this structure provides a cost-effective, high quality model to support primary care physicians in safe pre enging practices.

What are the real advantages of repurposing traditional anticoagulation clinic model to $+$

als as OAT medication management clinics? Addiction, unlike diseases warranting anticoagulation, is highly stigmatized, and these clinics would also need to 定 
work towards overcoming stigma-related barriers. In order to be successful, medication management clinics would have to move beyond just managing medication dosing and drug monitoring protocols, and focus on offering multiple patient-centered benefits with resspect to providing an integrated approach to managing addiction. First, these clinics coulder a less stigmatizing environment where patients are more likely to engage in care relative to traditional OAT clinics in specialty addictions clinics. Second, the pha magist/nurse leaders of the clinic could offer patients more dedicated time to address thein cerns with respect to their addiction and building trust with the medical team. Finally this multidisciplinary approach could provide more dedicated time for patient education and behavioral counseling to encourage adherence to medication regimens whare not be possible by primary care physicians who are practising alone. (ర)

There is precedent for using a similar multi-disciplinary team structure in the setting of nrarraging long-term opioid prescriptions. Researchers at the Philadelphia Veterans Affairs Medical Center tested a nurse practitioner- and clinical pharmacist-led model, aideroy a multi-specialty pain team, to help support primary care providers follow opioid_nrescribing guidelines for patients with non-cancer chronic pain(5). After two years, the model had increased primary care provider satisfaction, saved pharmacy costs, eased medication misuse(5)(4). 
Although OAT management for patients with substance use disorders is not completely analogous to opiate management for patients with chronic pain, there are similar requirements in terms of a need for multi-specialty care (e.g., mental/behavioral health, pinnary care, and pain management), stigmatization, and possessing a need to monitor for adoution misuse and diversion. In the OAT medication management model, the - clinics could also play a critical role within health systems of identifying and treating patenth with chronic pain who have developed a co-occurring opioid use disorder.

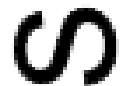

Sueaceful innovation often relies on re-inventing prior solutions and learning from other disciplines. When it comes to OAT, we should consider capitalizing on the infrastructure and once that exists within anticoagulation clinics, especially as their demand to ma varfarin decreases. These clinics have the potential to foster an environment of suppert and empathy where patients are going to heal rather than be chastised for their autuctron. This inventive re-interpretation of an existing model has the potential to incuease access to high quality care, lower health care costs, provide patient-centered car any allow more time for primary care physicians to manage diseases rather than druge We are in dire need of a readily available, effective solution to help curtail opioid related geaths. OAT medication management clinics in primary care may be the answer.

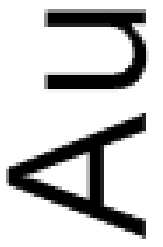


Acknowledgements: The authors would like to thank Vineet Chopra, MD, MSc for his valuable editorial comments. The authors have no conflicts of interest.

\footnotetext{
1. World Health Organization. How to improve Opioid Substitution Therapy impremptation.

htt w.euro.who.int/ data/assets/pdf file/0015/241341/How-to-improveQpioid_Substitution-Therapy-implementation.pdf?ua=1: 2014 January 2014.

2. McNeely J, Drucker E, Hartel D, Tuchman E. Office-based methadone prescribing: acceptance by inner-city practitioners in New York. Journal of urban he (th ? pulletin of the New York Academy of Medicine. 2000;77(1):96-102.

3. Chiquette E, Amato MG, Bussey HI. Comparison of an anticoagulation clinic with 9 al medical care: anticoagulation control, patient outcomes, and health care cos chives of internal medicine. 1998;158(15):1641-7.

4. Walley AY, Alperen JK, Cheng DM, Botticelli M, Castro-Donlan C, Samet JH, et al. Offic -based management of opioid dependence with buprenorphine: clinical practices and barriers. Journal of general internal medicine. 2008;23(9):1393-8.

5. Wiedemer NL, Harden PS, Arndt IO, Gallagher RM. The opioid renewal clinic: a primaty care, managed approach to opioid therapy in chronic pain patients at risk for outance abuse. Pain medicine (Malden, Mass). 2007;8(7):573-84.
}
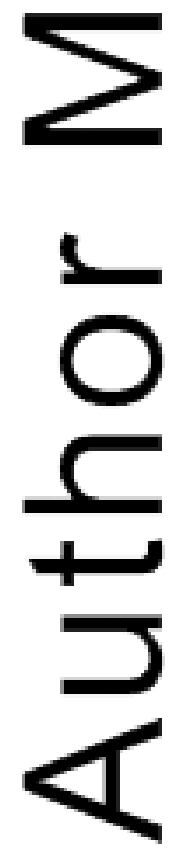